\title{
A regularity result for a class of non-uniformly elliptic operators
}

\author{
Fausto Ferrari and Giulio Galise
}

\begin{abstract}
We obtain an explicit Hölder regularity result for viscosity solutions of a class of second order fully nonlinear equations led by operators that are neither convex/concave nor uniformly elliptic.
\end{abstract}

Mathematics Subject Classification. 35J60, 35B65, 35D40.

Keywords. Viscosity solutions, Fully nonlinear partial differential equations, Non-uniformly elliptic operators.

1. Introduction. This note deals with the Hölder continuity issue of solutions of degenerate elliptic equations of the form

$$
\mathcal{M}_{\mathbf{a}}\left(D^{2} u\right)=f(x) \quad \text { in } \Omega,
$$

where $\Omega \subset \mathbb{R}^{N}$ is a domain, $f \in C(\Omega)$, and $\mathcal{M}_{\mathbf{a}}$ is the weighted partial trace operator defined, for any symmetric matrix $X$, by the formula

$$
\mathcal{M}_{\mathbf{a}}(X)=\sum_{i=1}^{N} a_{i} \lambda_{i}(X)
$$

In (1.2), $\lambda_{1}(X) \leq \cdots \leq \lambda_{N}(X)$ are the ordered eigenvalues of $X \in \mathbb{S}^{N}$, where $\mathbb{S}^{N}$ denotes the space of $N \times N$ real symmetric matrices, and $\mathbf{a}=\left(a_{1}, \ldots, a_{N}\right)$ is such that $a_{i} \geq 0$ for any $i=1, \ldots, N$. It is plain that $\mathcal{M}_{\mathbf{a}}$ reduces to the classical Laplace operator when $\mathbf{a}=(1, \ldots, 1)$ and that it falls out the class of uniformly elliptic operators as soon as $a_{i}=0$ for some $i=1, \ldots, N$.

Fausto Ferrari was partially supported by INDAM-GNAMPA 2019 project: Proprietà di regolarità delle soluzioni viscose con applicazioni a problemi di frontiera libera and INDAMGNAMPA 2020 project: Metodi di viscosità e applicazioni a problemi non lineari con debole ellitticità. Giulio Galise was partially supported by INDAM-GNAMPA 2019 project: Problemi differenziali per operatori fully nonlinear fortemente degeneri and INDAM-GNAMPA 2020 project: Problemi asintotici per EDP nonlineari e Mean Field Games. 
Such operators include, as particular cases, significant examples of degenerate operators, for instance,

$$
\mathcal{P}_{k}^{-}(X)=\sum_{i=1}^{k} \lambda_{i}(X) \quad \text { and } \quad \mathcal{P}_{k}^{+}(X)=\sum_{i=1}^{k} \lambda_{N-k+i}(X),
$$

which arise in the study of various geometric and elliptic problems, see, e.g., [1, $2,9-11,16-18,22,23]$, as well as the operators $\lambda_{k}(X)$, for some $k \in\{1, \ldots, N\}$, whose interest has been developed in the framework of differential games theory, see [3-6].

In [15], the authors studied qualitative properties of solutions of (1.1) under the further assumption that $a_{1}>0$ and $a_{N}>0$, having in mind as prototype the Isaacs operator

$$
\lambda_{1}(X)+\lambda_{N}(X)=\min _{|\xi|=1} \max _{|\eta|=1}(\langle X \xi, \xi\rangle+\langle X \eta, \eta\rangle),
$$

which is neither uniformly elliptic nor convex/concave (in dimension $N \geq 3$ ). Among other results, they in particular obtained an Alexandov-BakelmanPucci (ABP) type inequality following the scheme of the proof showed in [7], starting from the fact that (see [7, Section 2.2] for the notation) if $u$ is a viscosity solution of (1.1), then

$$
u \in \underline{\mathcal{S}}\left(\frac{a_{*}}{N-1},|\mathbf{a}|_{\infty}, f\right) \cap \overline{\mathcal{S}}\left(\frac{a_{*}}{N-1},|\mathbf{a}|_{\infty}, f\right),
$$

where from now on we denote $a_{*}=\min \left\{a_{1}, a_{N}\right\},|\mathbf{a}|_{1}=a_{1}+\cdots+a_{N}$, and $|\mathbf{a}|_{\infty}=\max \left\{a_{1}, \ldots, a_{N}\right\}$. As a byproduct, they obtain, in the same way as in the uniformly elliptic case, that viscosity solutions of $(1.1)$ are $C_{\mathrm{loc}}^{0, \alpha}(\Omega)$, where $\alpha \in(0,1)$, which is not explicitly known, depends on the constant that appears in the ABP estimate. They did not obtain any further result about a possible lower bound on $\alpha$ or, possibly, a sharper result about the regularity of solutions due to the lack of structure in the nonlinear equation.

The goal of this note is to provide an explicit lower bound for $\alpha$, only depending on $a_{1}$ and $a_{N}$ which therefore are assumed to be both positive. Let us point out that our result does not apply to $\mathcal{P}_{k}^{ \pm}$when $k<N$ since in these cases $a_{1}$ or $a_{N}$ are zero. Nevertheless, some regularity results concerning such operators, in particular for $k=1$, can be found in [19] and [2, Propositions 3.1-3.2]-[15, Theorem 1.4]. Applying the Ishii-Lions approach to the problem (see $[20]$ ), we manage to prove that viscosity solutions of $(1.1)$ are $C_{\text {loc }}^{0, \beta}(\Omega)$, where

$$
\beta=1-\frac{a_{1}+a_{N}}{\left(\sqrt{a_{1}}+\sqrt{a_{N}}\right)^{2}} .
$$

From this, we infer that $\alpha \geq \beta$ and, concerning the main example (1.3), we in particular obtain that $\alpha \geq \frac{1}{2}$. It is worth to point out that the fundamental assumption in the strategy of Ishii-Lions, in order to prove the Lipschitz continuity of solutions, is the uniformly ellipticity of the equation which clearly is outside our setting. Nevertheless, using the assumption $a_{1}>0$ and $a_{N}>0$, we are still able to detect some useful information encoded in the structure 
of the operator, so leading to the $\beta$-regularity of solutions, where $\beta$, defined in (1.5), is strictly less than one. In addition, this approach can be applied to a larger class of operators with first order terms. Thus, for stating our main result, we introduce the class of the equations we are going to consider. Let

$$
\mathcal{M}_{\mathbf{a}}\left(D^{2} u\right)+H(\nabla u)=f(x) \text { in } \Omega,
$$

where $\Omega \subseteq \mathbb{R}^{N}$ is a domain, $f$ is continuous in $\Omega$, and $\mathcal{M}_{\mathbf{a}}$ is the fully nonlinear operator that we have introduced in (1.2).

Our assumptions are:

(H1) $\mathcal{M}_{\mathbf{a}} \in \mathcal{A}=\left\{\mathcal{M}_{\mathbf{a}}(X):=\sum_{i=1}^{N} a_{i} \lambda_{i}(X): a_{i} \geq 0, i=1, \ldots, N, a_{1}>\right.$ $\left.0, a_{N}>0, X \in \mathbb{S}^{N}\right\}$.

(H2) $H \in C\left(\mathbb{R}^{N}\right)$ and there exists a nonnegative constant $C_{H}$ such that

$$
|H(p+q)-H(p)| \leq C_{H}(1+|p|+|q|)|q|
$$

for every $p, q \in \mathbb{R}^{N}$.

A typical example of $H$ satisfying (1.7) is $H(p)=A|p|^{2}+B|p|^{\tau}$, where $\tau \in[0,2]$ and $A, B \in \mathbb{R}$. Although we shall allow $H$ to have a quadratic growth in the gradient variable, the prototype equation to be kept in mind is still the one obtained when $H \equiv 0$, e.g.,

$$
a_{1} \lambda_{1}\left(D^{2} u\right)+a_{N} \lambda_{N}\left(D^{2} u\right)=f(x) \quad \text { in } \Omega,
$$

with $a_{1}, a_{N}>0$.

Now, we are in position to state our main result.

Theorem 1.1. Let $u \in C(\Omega)$ be a viscosity solution of (1.6). If (H1)-(H2) hold, then

$$
u \in C_{l o c}^{0, \beta}(\Omega), \quad \beta=1-\frac{a_{1}+a_{N}}{\left(\sqrt{a_{1}}+\sqrt{a_{N}}\right)^{2}},
$$

and the following estimate holds: for any $\omega \subset \subset \omega^{\prime} \subset \subset \Omega$, one has

$$
\|u\|_{C^{0, \beta}(\omega)} \leq C=C\left(a_{1}, a_{N}, \operatorname{dist}\left(\omega, \omega^{\prime}\right), C_{H},\|u\|_{L^{\infty}\left(\omega^{\prime}\right)},\|f\|_{L^{\infty}\left(\omega^{\prime}\right)}\right) .
$$

The main consequence of Theorem 1.1 is a lower bound of the expected regularity of viscosity solutions to a large class of operators that are not uniformly elliptic. We point out that very few results are known about the sharp regularity of solutions to fully nonlinear equations that are not convex/concave and that are not uniformly elliptic. In particular, we recall the fundamental result [21]. Concerning the regularity issues of viscosity solutions of degenerate equations closely related to ours, we refer to $[8,13-15]$.

We conclude this introduction by pointing out that if we drop the assumption (H1), in the sense that $a_{1}=a_{N}=0$, then there exist viscosity solutions of

$$
a_{2} \lambda_{2}\left(D^{2} u\right)+\cdots+a_{N-1} \lambda_{N-1}\left(D^{2} u\right)=0 \text { in } B_{1},
$$

which do not belong to any $C_{\mathrm{loc}}^{0, \alpha}\left(B_{1}\right)$ for $\alpha \in(0,1)$, even if $a_{i}>0$ for any $i=2, \ldots, N-1$. We present a simple example at the end of this note. 
2. Hölder regularity. We start with the following elementary lemma.

Lemma 2.1. Let $\delta>0$ and let $A, B, C, D$ be nonnegative constants such that $A \in(0,1)$ and $C>0$. Then there exists $\varphi \in C^{2}((0, \delta]) \cap C([0, \delta])$, depending on $A, B, C, D, \delta$, which is a positive solution of

$$
\varphi^{\prime \prime}(r)+\left(\frac{A}{r}+B\right) \varphi^{\prime}(r)=-C, \quad r \in(0, \delta],
$$

and satisfies the following conditions:

$$
\begin{aligned}
& \varphi^{\prime}(r)>0 \quad \text { and } \quad \varphi^{\prime \prime}(r)<0 \quad \text { for any } r \in(0, \delta], \\
& \varphi^{\prime \prime}(r)-\frac{\varphi^{\prime}(r)}{r} \leq-C \text { for any } r \in(0, \delta], \\
& \varphi(\delta) \geq D, \\
& \sup _{0<r \leq \delta} \frac{\varphi(r)}{r^{1-A}}<+\infty .
\end{aligned}
$$

Proof. By a straightforward computation, for any $K \in \mathbb{R}$, the function

$$
\varphi(r)=\int_{0}^{r} \psi(s) d s \quad \text { where } \quad \psi(s)=\frac{e^{-B s}}{s^{A}}\left(K-C \int_{0}^{s} t^{A} e^{B t} d t\right)
$$

is a solution of (2.1). Pick $K=K(A, B, C, D, \delta)$ such that $\psi(\delta)=\frac{D}{\delta}$. Hence $\varphi^{\prime}(r)>0$ for $r \in(0, \delta]$ and, since $\varphi(0)=0$, then $\varphi(r)>0$ for any $r \in(0, \delta]$. Moreover, just using the equation (2.1), we infer that (2.2) holds. Condition (2.3) easily follows by (2.2) and again using (2.1). Since $\psi$ is a decreasing function in $(0, \delta]$, we have

$$
\varphi(\delta)=\int_{0}^{\delta} \psi(s) d s \geq \delta \psi(\delta)=D
$$

by the choice of $K$. This shows (2.4). To conclude, it is sufficient to observe that, for any $r \in(0, \delta]$,

$$
\varphi(r) \leq K \int_{0}^{r} \frac{1}{s^{A}} d s=\frac{K}{1-A} r^{1-A}
$$

Proof of Theorem 1.1. Take $\delta>0$ small enough such that $\omega_{2 \delta}=\left\{x \in \mathbb{R}^{N}\right.$ : $\operatorname{dist}(x, \omega)<2 \delta\} \subset \omega^{\prime}$. Fix $z \in \omega$ and let

$$
\Delta_{z}=\{(x, y) \in \Omega \times \Omega:|x-y|<\delta,|x-z|<\delta\} .
$$

Note that if $(x, y) \in \Delta_{z}$, then both $x$ and $y$ belong in particular to $\omega^{\prime}$. For $(x, y) \in \bar{\Delta}_{z}$, let

$$
\phi(x, y)=u(x)-u(y)-\varphi(|x-y|)-L|x-z|^{2},
$$


where $\varphi(r)$ is, for $r \in[0, \delta]$, the function provided by Lemma 2.1 and depending on the parameters $A, B, C, D$. Let $A=1-\beta \in(0,1)$. We claim that for an appropriate choice of $B, C, D$ and $L$, then

$$
\max _{(x, y) \in \bar{\Delta}_{z}} \phi(x, y) \leq 0 .
$$

This will imply the desired result, taking first $x=z$, then making $z$ vary and using (2.5).

Set

$$
\begin{aligned}
L & =\frac{2\|u\|_{L^{\infty}\left(\omega^{\prime}\right)}}{\delta^{2}}, \\
D & =2\|u\|_{L^{\infty}\left(\omega^{\prime}\right)}, \\
C & =\frac{2\left(L\left(|\mathbf{a}|_{1}+C_{H} \delta(1+2 L \delta)\right)+\|f\|_{L^{\infty}\left(\omega^{\prime}\right)}+1\right)}{\left(\sqrt{a_{1}}+\sqrt{a_{N}}\right)^{2}}, \\
B & =\frac{2 L \delta C_{H}}{\left(\sqrt{a_{1}}+\sqrt{a_{N}}\right)^{2}} .
\end{aligned}
$$

By contradiction, we suppose that (2.6) does not hold. Let $(\hat{x}, \hat{y}) \in \bar{\Delta}_{z}$ be such that

$$
\max _{(x, y) \in \bar{\Delta}_{z}} \phi(x, y)=\phi(\hat{x}, \hat{y})>0 .
$$

By (2.8), it is clear that $\hat{x} \neq \hat{y}$. Moreover, using (2.7), we exclude that $|\hat{x}-\hat{y}|=\delta$ or $|\hat{x}-z|=\delta$. Hence $(\hat{x}, \hat{y}) \in \Delta_{z}$. By a standard result in theory of viscosity solutions, see [12, Theorem 3.2 and Remark 3.8], for any $\varepsilon>0$, there exist matrices $X_{\varepsilon}, Y_{\varepsilon} \in \mathbb{S}^{N}$ such that

$$
\begin{aligned}
& \left(\nabla \varphi(|\hat{x}-\hat{y}|)+2 L(\hat{x}-z), X_{\varepsilon}+2 L I\right) \in \bar{J}^{2,+} u(\hat{x}), \\
& \left(\nabla \varphi(|\hat{x}-\hat{y}|), Y_{\varepsilon}\right) \in \bar{J}^{2,-} u(\hat{y}),
\end{aligned}
$$

and

$$
\left(\begin{array}{cc}
X_{\varepsilon} & 0 \\
0 & -Y_{\varepsilon}
\end{array}\right) \leq\left(\begin{array}{rr}
\Theta_{\varepsilon} & -\Theta_{\varepsilon} \\
-\Theta_{\varepsilon} & \Theta_{\varepsilon}
\end{array}\right),
$$

where $I \in \mathbb{S}^{N}$ is the identity matrix and $\Theta_{\varepsilon} \in \mathbb{S}^{N}$ is given by

$$
\begin{aligned}
\Theta_{\varepsilon}= & \varphi^{\prime \prime}(|\hat{x}-\hat{y}|)\left(1+2 \varepsilon \varphi^{\prime \prime}(|\hat{x}-\hat{y}|)\right) P \\
& +\frac{\varphi^{\prime}(|\hat{x}-\hat{y}|)}{|\hat{x}-\hat{y}|}\left(1+2 \varepsilon \frac{\varphi^{\prime}(|\hat{x}-\hat{y}|)}{|\hat{x}-\hat{y}|}\right)(I-P)
\end{aligned}
$$

where $P=\frac{\hat{x}-\hat{y}}{|\hat{x}-\hat{y}|} \otimes \frac{\hat{x}-\hat{y}}{|\hat{x}-\hat{y}|}$. Note that the eigenvalues of $\Theta_{\varepsilon}$ are $\varphi^{\prime \prime}(|\hat{x}-\hat{y}|)(1+$ $\left.2 \varepsilon \varphi^{\prime \prime}(|\hat{x}-\hat{y}|)\right)$, which is simple, and $\frac{\varphi^{\prime}(|\hat{x}-\hat{y}|)}{|\hat{x}-\hat{y}|}\left(1+2 \varepsilon \frac{\varphi^{\prime}(|\hat{x}-\hat{y}|)}{|\hat{x}-\hat{y}|}\right)$ with multiplicity $N-1$. In view of (2.3), we can assume that, for $\varepsilon$ sufficiently small, one has

$$
\lambda_{1}\left(\Theta_{\varepsilon}\right)=\varphi^{\prime \prime}(|\hat{x}-\hat{y}|)\left(1+2 \varepsilon \varphi^{\prime \prime}(|\hat{x}-\hat{y}|)\right)
$$


and that

$$
\lambda_{2}\left(\Theta_{\varepsilon}\right)=\cdots=\lambda_{N}\left(\Theta_{\varepsilon}\right)=\frac{\varphi^{\prime}(|\hat{x}-\hat{y}|)}{|\hat{x}-\hat{y}|}\left(1+2 \varepsilon \frac{\varphi^{\prime}(|\hat{x}-\hat{y}|)}{|\hat{x}-\hat{y}|}\right) .
$$

Using (2.9)-(2.10), and the equation (1.6), we then obtain

$$
\begin{aligned}
-2\|f\|_{L^{\infty}\left(\omega^{\prime}\right)} \leq a_{1} & \lambda_{1}\left(X_{\varepsilon}\right)+a_{N} \lambda_{N}\left(X_{\varepsilon}\right)-a_{1} \lambda_{1}\left(Y_{\varepsilon}\right)-a_{N} \lambda_{N}\left(Y_{\varepsilon}\right) \\
& +\sum_{i=2}^{N-1} a_{i}\left(\lambda_{i}\left(X_{\varepsilon}\right)-\lambda_{i}\left(Y_{\varepsilon}\right)\right)+2 L|\mathbf{a}|_{1} \\
& +H(\nabla \varphi(|\hat{x}-\hat{y}|)+2 L(\hat{x}-z))-H(\nabla \varphi(|\hat{x}-\hat{y}|)) .
\end{aligned}
$$

Since $X_{\varepsilon} \leq Y_{\varepsilon}$ and $a_{i} \geq 0$, then using (1.7) and (2.2), we have

$$
\begin{gathered}
-2\|f\|_{L^{\infty}\left(\omega^{\prime}\right)} \leq a_{1} \lambda_{1}\left(X_{\varepsilon}\right)+a_{N} \lambda_{N}\left(X_{\varepsilon}\right)-a_{1} \lambda_{1}\left(Y_{\varepsilon}\right)-a_{N} \lambda_{N}\left(Y_{\varepsilon}\right) \\
+2 L|\mathbf{a}|_{1}+2 L \delta C_{H}\left(1+\varphi^{\prime}(|\hat{x}-\hat{y}|)+2 L \delta\right) .
\end{gathered}
$$

In order to reach a contradiction, we now estimate the right hand side of (2.12) using the inequality

$$
\left(\begin{array}{cc}
X_{\varepsilon} & 0 \\
0 & -Y_{\varepsilon}
\end{array}\right)\left(\begin{array}{c}
v \\
w
\end{array}\right) \cdot\left(\begin{array}{c}
v \\
w
\end{array}\right) \leq\left(\begin{array}{rr}
\Theta_{\varepsilon} & -\Theta_{\varepsilon} \\
-\Theta_{\varepsilon} & \Theta_{\varepsilon}
\end{array}\right)\left(\begin{array}{c}
v \\
w
\end{array}\right) \cdot\left(\begin{array}{c}
v \\
w
\end{array}\right) \quad \forall v, w \in \mathbb{R}^{N}
$$

and choosing $v, w$ in a suitable way. With the choice

$$
v=\sqrt{a_{1}} \frac{\hat{x}-\hat{y}}{|\hat{x}-\hat{y}|}, \quad w=-\sqrt{a_{N}} \frac{\hat{x}-\hat{y}}{|\hat{x}-\hat{y}|},
$$

then (2.13) yields

$$
\begin{aligned}
a_{1} \lambda_{1}\left(X_{\varepsilon}\right)-a_{N} \lambda_{N}\left(Y_{\varepsilon}\right) & \leq a_{1} X_{\varepsilon} \frac{\hat{x}-\hat{y}}{|\hat{x}-\hat{y}|} \cdot \frac{\hat{x}-\hat{y}}{|\hat{x}-\hat{y}|}-a_{N} Y_{\varepsilon} \frac{\hat{x}-\hat{y}}{|\hat{x}-\hat{y}|} \cdot \frac{\hat{x}-\hat{y}}{|\hat{x}-\hat{y}|} \\
& \leq\left(\sqrt{a_{1}}+\sqrt{a_{N}}\right)^{2} \Theta_{\varepsilon} \frac{\hat{x}-\hat{y}}{|\hat{x}-\hat{y}|} \cdot \frac{\hat{x}-\hat{y}}{|\hat{x}-\hat{y}|} \\
& =\left(\sqrt{a_{1}}+\sqrt{a_{N}}\right)^{2} \varphi^{\prime \prime}(|\hat{x}-\hat{y}|)\left(1+2 \varepsilon \varphi^{\prime \prime}(|\hat{x}-\hat{y}|)\right)
\end{aligned}
$$

On the other hand, taking

$$
v=\sqrt{a_{N}} \xi, \quad w=0,
$$

where $|\xi|=1$ and $X_{\varepsilon} \xi=\lambda_{N}\left(X_{\varepsilon}\right) \xi$, we have

$$
a_{N} \lambda_{N}\left(X_{\varepsilon}\right) \leq a_{N} \Theta_{\varepsilon} \xi \cdot \xi \leq a_{N} \frac{\varphi^{\prime}(|\hat{x}-\hat{y}|)}{|\hat{x}-\hat{y}|}\left(1+2 \varepsilon \frac{\varphi^{\prime}(|\hat{x}-\hat{y}|)}{|\hat{x}-\hat{y}|}\right) .
$$

In a similar way, we also obtain that

$$
-a_{1} \lambda_{1}\left(Y_{\varepsilon}\right) \leq a_{1} \Theta_{\varepsilon} \xi \cdot \xi \leq a_{1} \frac{\varphi^{\prime}(|\hat{x}-\hat{y}|)}{|\hat{x}-\hat{y}|}\left(1+2 \varepsilon \frac{\varphi^{\prime}(|\hat{x}-\hat{y}|)}{|\hat{x}-\hat{y}|}\right) .
$$


Putting together (2.12), (2.14)-(2.16), we infer that

$$
\begin{aligned}
-2\|f\|_{L^{\infty}\left(\omega^{\prime}\right)} \leq & \left(\sqrt{a_{1}}+\sqrt{a_{N}}\right)^{2} \varphi^{\prime \prime}(|\hat{x}-\hat{y}|)+\left(\frac{a_{1}+a_{N}}{|\hat{x}-\hat{y}|}+2 L \delta C_{H}\right) \varphi^{\prime}(|\hat{x}-\hat{y}|) \\
& +2 L\left(|\mathbf{a}|_{1}+C_{H} \delta(1+2 L \delta)\right) \\
& +2 \varepsilon\left[\left(\sqrt{a_{1}}+\sqrt{a_{N}}\right)^{2}\left(\varphi^{\prime \prime}(|\hat{x}-\hat{y}|)\right)^{2}+\left(a_{1}+a_{N}\right)\left(\frac{\varphi^{\prime}(|\hat{x}-\hat{y}|)}{|\hat{x}-\hat{y}|}\right)^{2}\right] .
\end{aligned}
$$

By (2.7) and Lemma 2.1, the function $\varphi(r)$ is a solution, for $r \in(0, \delta]$, of the ordinary differential equation

$$
\begin{aligned}
\left(\sqrt{a_{1}}+\sqrt{a_{N}}\right)^{2} \varphi^{\prime \prime}(r) & +\left(\frac{a_{1}+a_{N}}{r}+2 L \delta C_{H}\right) \varphi^{\prime}(r)= \\
& =-2\left(L\left(|\mathbf{a}|_{1}+C_{H} \delta(1+2 L \delta)\right)+\|f\|_{L^{\infty}\left(\omega^{\prime}\right)}+1\right) .
\end{aligned}
$$

Coupling (2.17)-(2.18), then

$$
1 \leq \varepsilon\left[\left(\sqrt{a_{1}}+\sqrt{a_{N}}\right)^{2}\left(\varphi^{\prime \prime}(|\hat{x}-\hat{y}|)\right)^{2}+\left(a_{1}+a_{N}\right)\left(\frac{\varphi^{\prime}(|\hat{x}-\hat{y}|)}{|\hat{x}-\hat{y}|}\right)^{2}\right]
$$

leading to a contradiction for $\varepsilon$ small enough.

Remark 2.2. We note that in the case $H \equiv 0$, the function $\varphi(r)$ used in the proof of Theorem 1.1 is more explicit, in fact it is given by $\varphi(r)=A r^{\beta}-B r^{2}$ for a suitable choice of $A, B>0$.

2.1. Lack of regularity. Let $N \geq 3$ and consider the equation

$$
a_{2} \lambda_{2}\left(D^{2} u\right)+\cdots+a_{N-1} \lambda_{N-1}\left(D^{2} u\right)=0 \text { in } B_{1} .
$$

We are going to exhibit a continuous function $u$ which is a solution of (2.19) for any $a_{i} \geq 0$ and $i=2, \ldots, N-1$, but which does not belong to $C_{\text {loc }}^{0, \alpha}\left(B_{1}\right)$ for any possible choice of $\alpha \in(0,1]$.

Let $f:(-1,1) \mapsto \mathbb{R}$ be the function

$$
f(t)=\left\{\begin{array}{cl}
\frac{1}{2-\log |t|} & \text { if } t \neq 0 \\
0 & \text { if } t=0
\end{array}\right.
$$

and consider it as a function of $N$ variables just by setting $u(x)=f\left(x_{1}\right)$ for $x \in B_{1}$. It is clear that $u \in C\left(B_{1}\right)$ but $u \notin C_{\text {loc }}^{0 . \alpha}\left(B_{1}\right)$ for any $\alpha \in(0,1]$. We claim that $u$ is a viscosity solution of (2.19).

The function $u$ is smooth for $x \in B_{1} \backslash\left\{x \in B_{1}: x_{1}=0\right\}$ and

$$
D^{2} u(x)=\operatorname{diag}\left(f^{\prime \prime}\left(x_{1}\right), 0, \ldots, 0\right) .
$$

Since $f^{\prime \prime}(t) \leq 0$ for any $t \in(-1,1) \backslash\{0\}$, we infer that $u$ is in fact a classical solution of (2.19) in the set $B_{1} \backslash\left\{x \in B_{1}: x_{1}=0\right\}$. Now we prove that $u$ satisfies (in viscosity sense) the equation (2.19) also in $\left\{x \in B_{1}: x_{1}=0\right\}$. For $x \in \mathbb{R}^{N}$ such that $x_{1}=0$, we adopt the notation $x=\left(0, x^{\prime}\right)$ with $x^{\prime} \in \mathbb{R}^{N-1}$. Let $x_{0}=\left(0, x_{0}^{\prime}\right) \in B_{1}$. Since there are no test functions $\phi \in C^{2}\left(B_{1}\right)$ touching $u$ from above at $x_{0}$, we infer that $u$ is a viscosity subsolution of (2.19). As 
far as the supersolution property is concerned, it is sufficient to prove that if $\phi \in C^{2}\left(B_{1}\right)$ is such that

$$
0=u\left(0, x_{0}^{\prime}\right)=\phi\left(0, x_{0}^{\prime}\right) \text { and } u(x) \geq \phi(x) \quad \forall x \in B_{1},
$$

then $\lambda_{N-1}\left(D^{2} \phi\left(0, x_{0}^{\prime}\right)\right) \leq 0$. Set $\psi\left(x^{\prime}\right)=\phi\left(0, x^{\prime}\right)$ for $\left|x^{\prime}\right|<1$. From $(2.20)$, we deduce that $\psi\left(x^{\prime}\right)$ attains its maximum at $x_{0}^{\prime}$. Hence

$$
\left\langle D^{2} \psi\left(x_{0}^{\prime}\right) v, v\right\rangle \leq 0 \quad \forall v \in \mathbb{R}^{N-1} .
$$

Using the Courant-Fischer formula

$$
\lambda_{N-1}\left(D^{2} \phi\left(0, x_{0}^{\prime}\right)\right)=\min _{\operatorname{dim} W=N-1} \max _{\substack{w \in W \\|w|=1}}\left\langle D^{2} \phi\left(0, x_{0}^{\prime}\right) w, w\right\rangle,
$$

with the particular choice of $W=\left\{(0, v): v \in \mathbb{R}^{N-1}\right\}$, and $(2.21)$, we then obtain

$$
\lambda_{N-1}\left(D^{2} \phi\left(0, x_{0}^{\prime}\right)\right) \leq \max _{\substack{v \in \mathbb{R}^{N-1} \\|v|=1}}\left\langle D^{2} \psi\left(x_{0}^{\prime}\right) v, v\right\rangle \leq 0 .
$$

This shows that $u(x)$ is a viscosity solution of (2.19), for any $a_{i} \geq 0$ and $i=2, \ldots, N-1$.

We end this note by pointing out that the regularity issue for solutions of (1.1) in the case $a_{1}=0$ and $a_{N}>0$ (or $a_{1}>0$ and $a_{N}=0$ ) is an open problem and only partial results are known: Lipschitz regularity for $\mathcal{P}_{1}^{ \pm}$, see [2], Hölder estimates for $\mathcal{M}_{\mathbf{a}}$ in the case of asymmetric distributions of weights concentrated on the smallest or on the largest eigenvalue, namely $a_{1}>a_{2}+\cdots+a_{N}$ or $a_{N}>a_{1}+\cdots+a_{N-1}$, see [15] and the references therein.

Open Access. This article is licensed under a Creative Commons Attribution 4.0 International License, which permits use, sharing, adaptation, distribution and reproduction in any medium or format, as long as you give appropriate credit to the original author(s) and the source, provide a link to the Creative Commons licence, and indicate if changes were made. The images or other third party material in this article are included in the article's Creative Commons licence, unless indicated otherwise in a credit line to the material. If material is not included in the article's Creative Commons licence and your intended use is not permitted by statutory regulation or exceeds the permitted use, you will need to obtain permission directly from the copyright holder. To view a copy of this licence, visit http://creativecommons. org/licenses/by/4.0/.

Publisher's Note Springer Nature remains neutral with regard to jurisdictional claims in published maps and institutional affiliations.

\section{References}

[1] Ambrosio, L., Soner, H.M.: Level set approach to mean curvature flow in arbitrary codimension. J. Differential Geom. 43, 693-737 (1996)

[2] Birindelli, I., Galise, G., Ishii, H.: A family of degenerate elliptic operators: maximum principle and its consequences Ann. Inst. H. Poincaré Anal. Non Lináire 35(2), 417-441 (2018) 
[3] Blanc, P., Esteve, C., Rossi, J.D.: The evolution problem associated with eigenvalues of the Hessian. J. Lond. Math. Soc. 102(3), 1293-1317 (2020)

[4] Blanc, P., Rossi, J.D.: Games for eigenvalues of the Hessian and concave/convex envelopes. J. Math. Pures Appl. 127, 192-215 (2019)

[5] Buckdahn, R., Cardaliaguet, P., Quincampoix, M.: Some recent aspects of differential game theory. Dyn. Games Appl. 1, 74-114 (2011)

[6] Buckdahn, R., Li, J.: Stochastic differential games and viscosity solutions of Hamilton-Jacobi-Bellman-Isaacs equations. SIAM J. Control Optim. 47(1), 444475 (2008)

[7] Caffarelli, L.A., Cabré, X.: Fully Nonlinear Elliptic Equations. American Mathematical Society Colloquium Publications, 43. American Mathematical Society, Providence, RI (1995)

[8] Capuzzo Dolcetta, I., Leoni, F., Porretta, A.: Hölder estimates for degenerate elliptic equations with coercive Hamiltonians. Trans. Amer. Math. Soc. 362(9), 4511-4536 (2010)

[9] Capuzzo Dolcetta, I., Leoni, F., Vitolo, A.: On the inequality $F\left(x, D^{2} u\right) \geq f(u)+$ $g(u)|D u|^{q}$. Math. Ann. 365(1-2), 423-448 (2016)

[10] Caffarelli, L.A., Li, Y.Y., Nirenberg, L.: Some remarks on singular solutions of nonlinear elliptic equations: I. J. Fixed Point Theory Appl. 5, 353-395 (2009)

[11] Caffarelli, L.A., Li, Y.Y., Nirenberg, L.: Some remarks on singular solutions of nonlinear elliptic equations: III: viscosity solutions, including parabolic operators. Comm. Pure Appl. Math. 66, 109-143 (2013)

[12] Crandall, M.G., Ishii, H., Lions, P.L.: User's guide to viscosity solutions of second order partial differential equations. Bull. Amer. Math. Soc. (NS) 27(1), 1-67 (1992)

[13] Ferrari, F.: An application of the theorem on sums to viscosity solutions of degenerate fully nonlinear equations. Proc. Roy. Soc. Edinburgh Sect. A 150(2), 975-992 (2020)

[14] Ferrari, F., Vecchi, E.: Hölder behavior of viscosity solutions of some fully nonlinear equations in the Heisenberg group. Topol. Methods Nonlinear Anal. 55(1), 227-242 (2020)

[15] Ferrari, F., Vitolo, A.: Regularity properties for a class of non-uniformly elliptic Isaacs operators. Adv. Nonlinear Stud. 20(1), 213-241 (2020)

[16] Han, F., Ma, X.-N., Wu, D.: The existence of $k$-convex hypersurface with prescribed mean curvature. Calc. Var. Partial Differential Equations 42(1-2), 43-72 (2011)

[17] Harvey, F.R., Lawson, H.B., Jr.: Existence, uniqueness and removable singularities for nonlinear partial differential equations in geometry. In: Surveys in Differential Geometry. Geometry and Topology, pp. 103-156. Surv. Differ. Geom., 18. Int. Press, Somerville, MA (2013)

[18] Harvey, F.R., Lawson, H.B., Jr.: Removable singularities for nonlinear subequations. Indiana Math. J. 63(5), 1525-1552 (2014) 
[19] Oberman, A., Silvestre, L.: The Dirichlet problem for the convex envelope. Trans. Amer. Math. Soc. 363(11), 5871-5886 (2011)

[20] Ishii, H., Lions, P.-L.: Viscosity solutions of fully nonlinear second-order elliptic partial differential equations. J. Differential Equations 83(1), 26-78 (1990)

[21] Nadirashvili, N., Vlăduţ, S.: Nonclassical solutions of fully nonlinear elliptic equations. Geom. Funct. Anal. 17(4), 1283-1296 (2007)

[22] Sha, J.-P.: p-convex Riemannian manifolds. Invent. Math. 83, 437-447 (1986)

[23] Wu, H.: Manifolds of partially positive curvature. Indiana Univ. Math. J. 36, 525-548 (1987)

FAusto FERRARI

Dipartimento di Matematica

Università di Bologna

Piazza di Porta S. Donato 5

Bologna

Italy

e-mail: fausto.ferrari@unibo.it

Giulio Galise

Dipartimento di Matematica Guido Castelnuovo

Sapienza Università di Roma

Piazzale Aldo Moro 5

Roma

Italy

e-mail: galise@mat.uniroma1.it

Received: 7 October 2021

Accepted: 21 January 2022. 